\title{
A Preliminary Investigation into the Use of an Imagery Assisted Virtual Reality Intervention in Sport
}

\author{
Lindsay Ross-Stewart ${ }^{1}$, Jeffrey Price ${ }^{2}$, Daniel Jackson ${ }^{3}$ and Christopher Hawkins ${ }^{4}$ \\ 1. Department of Applied Health, Southern Illinois University Edwardsville, Edwardsville IL 62026, USA \\ 2. Department of Information Technology Services, Southern Illinois University Edwardsville, Edwardsville IL 62026, USA \\ 3. Intercollegiate Athletics, McKendree University, Lebanon IL 62254, USA \\ 4. Anderson Hospital, Maryville IL 62062, USA
}

\begin{abstract}
This article explains an imagery assisted virtual reality psychological skills training program used with a NCAA Division I baseball team. This is the first time that imagery has been incorporated into a virtual reality program with the goal of increasing mental skills and strategies. Participants for this study were 27 NCAA baseball players. Each participant completed the Sport Imagery Ability Questionnaire and the Test of Performance Strategies Questionnaire at baseline and again after the winter season (2 months later). Results indicated an increase in skill, goals and mastery imagery ability as well as increases in the use of several skills and strategies in both practice and competition. This manuscript focuses on both the development of an Imagery Assisted Virtual Reality program as well as the outcomes of the program.
\end{abstract}

Key words: Imagery, mental training, virtual reality.

\section{Introduction}

From Bill Belichick to Carson Palmer, players and coaches at the highest level have begun to use and support virtual reality training in sport $[1,2]$. Sports Illustrated named Virtual Reality the 2015 Innovation of the Year [3]. Due to rapidly advancing VR technology, with Oculus Rift leading the way under the guidance of creator Palmer Luckey and investor Mark Zuckerberg [4], virtual reality is becoming a more accessible tool. To this point, people in the field of athletics have begun to explore how VR goggles can help with skill acquisition in elite sports. In 2015, articles were written in Bleacher Report [5], Sports Illustrated [3], NBC sports [2] and several other pop culture sport media outlets highlighting the role of VR in sport. However, at this time the use of VR in sport has been relegated to only the most elite teams.

Due to the ever-changing technology, virtual reality

Corresponding author: Lindsay Ross-Stewart, assistant professor, research field: sport psychology.
(VR) has been defined in many different ways over the last two decades, making a uniform definition impossible, however, regardless of the technology used, a key characteristic of VR has been the immersive experience it offers. For the purpose of this study, we have defined virtual reality as a first-person filmed, computer presented, immersive simulation of a real environment consisting of a monoscopic 360-degree image and stereo sound experienced through a head-mounted display. Immersive VR training can be many different things, but at the core is a requirement that a player watch film through the virtual reality goggles. The film can be taken via 360-degree cameras, so the players are able to move their head while wearing the goggles and see what they would see if they were in their true sport arena.

Recently, VR technology has been shown to be effective in a number of different health-related areas including; increasing occupational therapy compliance [6], accelerating physical rehabilitation [7, 8], speeding up the stroke rehabilitation process $[9,10]$ reducing 
symptoms of Parkinson's disease [11] distracting from pain [12] and used as exposure therapy for veterans with Post Traumatic Stress Disorder [13].

The use of virtual reality for sport is documented in several research papers. Using different types of VR technologies, researchers have shown the use of VR to positively affect decision-making in rugby [14], soccer skill [15], and decrease goalie reaction time in handball [16]. Athletes have used VR to experience going down a bobsledding track [17], and swimming [18] without actually performing the task. All these studies yielded positive results. It should be noted that these positive findings were all found before the newest technology allowed athletes to watch video of themselves in $\mathbf{3 6 0}$ via virtual reality headsets and goggles, making the potential impact of this new technology even more exciting.

Although the use of virtual reality for sport skill development is well documented we have yet to see virtual reality training make its way to smaller university teams or high school level teams. One reason may be that current VR technology may not be affordable for most programs. As such, there is a need to adapt the technology to make it affordable and approachable for teams and individual athletes to use. This is becoming increasingly possible now that VR goggles are readily available at affordable prices. Additionally, there is a need to tailor virtual reality training to focus on athlete's psychological skill development. At this time, the impact of VR training on athlete's psychological skills and techniques have not been addressed, even though, VR use in other health fields has shown to have a positive effect on psychological factors $[12,19]$.

To assess why Virtual Reality training may be successful for athletes physical and psychological skill development, it is pertinent to look to the research in the field of imagery as both allow a person to experience himself or herself completing a task without actually doing it. There are several hypotheses as to why imagery is effective, with particular attention on the reference [20] assertion that imaging a movement leads to the same neural activations as an actual movement. Holmes et al [21] along with several of their colleagues over the last 15 years have built on the idea of functional equivalency with the development of their PETTLEP approach to how imagery should be done by athletes [21-23]. Functional equivalency research postulates that you want the image to mimic the actions and feelings associated with actually doing the task, as much as possible. The PETTLEP model is an acronym for how an athlete can achieve a functionally equivalent imagery plan. Specifically, an image is considered functionally equivalent if it addresses seven areas. First, is the physical nature of the task, as evidenced by an athlete imaging in a way that attempts to replicate the physical nature of the task. The environment including all senses as well as the task they are imaging. Timing of the image should be the same as the time it would take to perform the actual task. Consideration of the learning that takes place as one develops their skill, as well as the emotion of the task are important to consider for equivalency. And finally, people should consider using an internal perspective for skill images and external, if desired, for other images [21]. It makes sense that seeing yourself completing your task, whether that task is swinging a bat, or staying calm in a stressful situation, in a virtual reality program would increase functional equivalency. The functional equivalency of VR may be an important component of how VR affects change in sport [14-18, 24].

Although the VR technology is constantly advancing, there are still components of the sport experience that are difficult to replicate via virtual reality. VR only focuses on the stimulus (e.g., seeing yourself swing a bat), while imagery allows for specific stimulus and response propositions (e.g., feeling confident with the swing) to be included in the experience. Therefore, it stands to reason, based on the theoretical underpinnings of functional equivalency, and research in both the field of imagery and VR that a VR program 
assisted with imagery would have the potential to affect psychology skill and strategy development even more than VR alone. Above and beyond that, imagery ability has been shown to significantly predict the ease with which a person will use imagery [25]. Therefore, developing ways to help athletes increase their imagery ability is an important component of any imagery intervention. Imagery is one of the most recommended psychological tools within the sport psychology literature, therefore, helping athletes develop imagery is of utmost importance [26]. Seeing oneself via VR, with opportunities to image built in to the VR protocol may be an effective way to increase imagery ability.

With this in mind, this paper presents the findings from a preliminary applied Imagery Assisted Virtual Reality protocol that focused on increasing psychological skill development (e.g., confidence, motivation) and psychological strategy use (e.g., imagery, relaxation) for a NCAA mid-size Division I baseball team. Of specific interest for this study was how Virtual Reality may affect imagery ability in athletes. Furthermore, this program assesses the value of using imagery in collaboration with the virtual reality training to develop an affordable, and effective imagery assisted VR intervention.

\section{Methods}

\subsection{Participants}

The 27 participants were all a part of one NCAA Division I baseball team. Seventeen hitters and ten pitchers were a part of the study. They ranged in age from 18-23 $(m=19.8)$. They had played baseball for an average of 14 years with an average of 1.65 years on their current team.

\subsection{Measures}

Sport Imagery Ability Questionnaire [27]. This questionnaire consists of 15 questions each of which are rated on a 1 (very hard to image) to 7 (very easy to image) Likert scale. The questions represent five subscales; skill imagery ability (e.g., refining a particular skill), strategy imagery ability (e.g., making up new plans/strategies in my head), goal imagery ability (e.g., myself winning a medal), affect imagery ability (e.g., the positive emptions I feel while doing my sport), and mastery imagery ability (e.g., staying positive after a setback). This questionnaire has been found to be both valid and reliable [25, 27].

Test of Performance Strategies [TOPS; 28]. TOPS is designed to assess an athletes use of psychological skills and strategies in both practice and competition through the use of 64 questions answered on a 1 (never use) - 5 (always use) Likert type scale. There are eight subscales assessing skills and strategies at practice and eight for competition. For both practice and competition TOPS assesses use of self-talk, goal setting, imagery, emotional control, automaticity, relaxation, and activation. For practice attentional control is assessed, while negative thinking is assessed for competition. TOPS is a well-established and validated tool [28].

\subsection{Procedure}

Due to the novel nature of the Imagery Assisted Virtual Reality Protocol used in this study, it is described in great detail with the hope of replication being possible in both applied and research settings.

\subsubsection{Step 1: Designing the Program}

This psychological skills program was intensive in program design. Specifically, the assistant coach of the baseball team, two technology specialists (also graduate students in an exercise and sport psychology program), and the team sport psychology consultant were all part of the design and implementation of this intervention. To start, the four members of the intervention team met to discuss the logistics of filming the players including: where the camera would be placed on each player, what type of camera would be worn, the film that would be most beneficial, and how players would gain access to the film. Of particular importance was the decision on what technology to use. After assessing multiple reviews on different products, 
the intervention team decided to use both 360 -film taken using the KODAK SP 360 and first-person immersive 180 film using a GoPro. ColorCross VR goggles were purchased for each player because they are effective and more affordable (\$16.00 each) than Oculus Rift or Samsung Galaxy Goggles. In total, the cameras and goggles cost the team less than $\$ 2000$.

When filming for VR purposes, there are two main options, Monoscopic and Stereoscopic. For this project, Monoscopic filming was used to create a single image presented to both eyes through the goggles. Stereoscopic video presents separate images to each eye from slightly different angles to create a three-dimensional (3D) effect. Stereoscopic 3D video requires two separate videos to be made which cuts the resolution in half. This, along with the difficulty filming with a larger stereoscopic camera setup, led us to adopt a monoscopic setup consistent with the vast majority of 360-degree videos currently available online.

During this phase, it was decided that first-person film, traditionally done for virtual reality training, as well as third-person film for those who preferred to watch themselves from an external perspective would be used. Regardless of perspective, all film would be watched through virtual reality Goggles for the immersive 3D experience. Once these decisions were made, three players (one pitcher, one hitter, one catcher) wore cameras at different angles using different head mounts so the team could test different film angles. They watched the film and gave their feedback on what angles they preferred.

\subsubsection{Step 2: Incorporation of Imagery}

Imagery has been described as "an experience that mimics real experience. We can be aware of 'seeing' an image, feeling movements as an image, or experiencing an image of smell, tastes or sounds without actually experiencing the real thing" [29]. Imagery is the most used psychological technique in the field of sport psychology [26] and has been shown to be effective for both physical and psychological development in sport [30, 31]. Recently research has indicated that video assisted imagery is an effective way to teach imagery, develop confidence and enhance performance [32, 33]. In discussing the implementation of the VR program, it was decided that film could be supplemented by having blank spaces where the athletes imaged an experience. This would do three things: (1) allow the VR to work as an aid in imagery development; (2) allow for some of the player's experience to be imaged instead of filmed and (3) allow for the addition of response propositions in the athletes experience. This led to the development of the imagery assisted virtual reality protocol (IAVR) used with the team.

Each imagery script was written with the intent to give the athlete an opportunity to see themselves be successful during their on deck swings/pre inning pitches, as well as to help each athlete solidify a pre swing/pitch routine that allowed them to control any psychological skills they wanted to achieve/maintain. To do this each athlete met with their assistant coach and the team sport psychology consultant to discuss their pre swing/pitch routine as well as what they felt they needed to work on the most as far as their psychological game. For example, an athlete who said they were nervous before their skill execution, had stimulus and response propositions built into their imagery that focused on being relaxed, and in the moment, while an athlete who wanted to focus on confidence had images focused on the stimulus of staying confident as they were on deck, and then seeing the response of executing successfully with in their image. Imagery scripts were written based on the guidelines of reference 34 on imagery script development and the PETTLEP approach [21].

Based on players' comments, and guided by the research on effective imagery protocols $[21-23,35,36]$, each player had a guided imagery script written for them. Players chose whether or not they wanted their imagery script to be recorded as audio playing during the imagery blank space of their VR film, or whether 
they simply wanted to have it written down, learn it and image it on their own.

\subsubsection{Step 3: Filming}

All players were filmed doing their skill (hitting/pitching). Each pitcher threw his three pitches, which allowed all pitchers to have video of all three pitches, and hitters to have video hitting off all pitches. Hitters who asked were able to get film off more than one pitcher.

Players were also filmed in non-skill specific scenarios meant to positively impact confidence and anxiety. Specifically, all players wore the camera (for a first-person perspective) walking out of the locker room, putting on their gloves, and then walking out to the batter's box during their walk up song. Players reported that watching these moments encouraged confidence while also helping them feel prepared. As these acts are not continuous, imagery was used to fill in the necessary spaces (see step 2). The first-person perspective video added a component in which athletes could feel the emotional experience of preparing to compete, through IAVR. For skill filming, a hitter, a catcher, and a pitcher were all wearing head mounted cameras at one time during filming. This allowed for skills to be filmed in both first person (player wearing the camera on a head mount) and third person (pitchers view for hitter, hitter and catchers view for pitcher) at the same time.

Unlike a dynamic sport like football or soccer in which the camera could be left on a player throughout a drill or scrimmage, baseball is much more static. For this reason, it was not possible to simply put a camera on a player and leave it on throughout a scrimmage or through an entire drill. Instead we had two filming days, one to get all skill footage necessary and one to get film for psychological development purposes.

2.3.4 Step 4: Player's Access to Virtual Reality Technology

Players had a meeting with the intervention team in which the IAVR intervention was reiterated (it had been described before filming). Each player was assigned their own VR goggles, and was shown how to use them. This included showing players how to download a 3D viewing app onto their phone. For iPhone, this was the mobile VR Station App. For Androids, it was Google Cardboard (now called VR Theatre). Players were then shown how to retrieve videos from the cloud, download them to the appropriate VR app on their cell phones, put their phones in the goggles, and use them. One of the features of the VR goggles is the ability to use eye direction to control the video (e.g., play, pause, rewind). Players were given time to practice these techniques with videos found on YouTube for a week while the intervention team edited the clips.

\subsubsection{Step 5: Editing}

First, the assistant baseball coach watched all the footage of the skill and assessed which clips showed the player being successful. The technology specialists then edited these clips into individual videos. Each player received his clips via the universities universal cloud storage system and was given a folder where he and the intervention team could access his clips.

\subsubsection{Step 6: Player Feedback/Imagery} Development

The players were asked for their opinions on the clips. Their feedback led to using 360-degree footage for motivational filming, and a combination of first and third person GoPro film for skill shots. Although initially surprising that the players did not want 360 film for the skill clips, players consistently indicated that they felt that having the 360 film was not beneficial as they do not look around when performing the skills being filmed, and liked the higher resolution film of the GoPro more for skill development. The clips chosen by a player were then made into one video sequence, that the player could play from start to finish. Each player also met with the sport psychology consultant and the assistant baseball coach to discuss what he wanted to image during the imagery spots on their video sequence (see step 2). For hitters, the first opportunity to image occurred when they were two 
people away from hitting, something they are encouraged to do in actual games. Having them image at the same time during the VR training allowed for functional equivalency to be increased. The second time that the video went blank for imagery was when they were to image themselves on deck, helping them solidify this pre-performance routine. For pitchers, they imaged their warm up routine.

Example sequence for hitters: (1) VR of walking out of the locker room seeing their team in the dugout (first-person 360 film). (2) Blank space in the video for participant imaging. In this case, hitter's image hitting off the opposing team pitcher. If chosen, audio of guided imagery script was playing. (3) VR of putting on their gloves (if applicable), grabbing their bat and walking to the edge of the dugout, (first-person, 360 film). (4) Blank space for imaging being on deck. If chosen, audio of guided imagery script playing. (5) VR of walking up to the batter's box, walk up music playing (first-person, 360 film). (6) VR of themselves taking good at bats. This could be making a good read for a walk, or making a good hit. Film always ended on their best swing. This film was a combination of first-person film taken via the hitter wearing the camera, and third-person film worn either by the catcher or pitcher. All film was taken from the GoPro.

\subsubsection{Step 7: Watching the Film Sequences}

Players were asked to watch the film one-two times a day, in their proper stance. At the beginning, one VR session was done at practice as a group to help players get in the habit of using the technique. In total, this was a time requirement of 12-15 minutes a day.

\subsubsection{Step 8: Follow Up on Program Effectiveness}

Three months after the players began the intervention they completed the SIAQ and TOPS. They were also asked to self-report on their use of the protocol. To identify any players who indicated they were using it, who really were not (social desirability) players were first asked to write out the two different imagery scripts that they imaged as part of the protocol. If a player had been doing the protocol this was something they should have easily been able to remember. If they had not done it, this was a difficult task. This was used as a reliability check to make sure all participants who said they complied with the protocol did so.

\section{Results}

Of the 27 participants in the intervention, 5 did not use the program. Of the 22 who used the program, the average times they used it a week was 10.54 times ( $\mathrm{min}$ $=1.5, \max =21$ ).

Due to the small number of participants who did not adhere to the program, this study was not able to statistically assess the difference in imagery ability and performance strategy use between those who participated and those who did not. It should also be noted that all five participants who did not adhere to the intervention were pitchers, all hitters adhered to the program. A preliminary assessment of means between those who completed the intervention 7 times or less a week (representing once a day) and those who did it more than 7 times a week was done, however there were no differences found. Therefore, all inferential statistics were run on the 22 participants who completed the intervention as one group.

Descriptive statistics including mean scores for the 16 TOPS subscales and the 5 Sport Imagery Ability Questionnaire Subscales at baseline and at time 2 are reported in Table 1. To assess the effectiveness of the protocol on the development of imagery ability repeated measures ANOVA's were run. A 2 (time) $\times 1$ (group) ANOVA indicated a significant mean difference between baseline and Time 2 for skill imagery $F(1,21)=4.91, P=0.04, \beta=0.56$. Mean scores indicate that participants skill imagery ability improved from baseline ( $m=5.62)$ to time $2(m=5.97)$. A 2 (time) $\times 1$ (group) ANOVA indicated a significant mean difference between baseline and Time 2 for goals imagery $F(1,21)=8.85, P=0.01, \beta=0.81$. Mean scores indicate that participants goals imagery ability improved from baseline $(m=5.77)$ to time $2(m=6.2)$. 
Table 1 Descriptive Statistics and alpha levels for TOPS and Sport Imagery Ability Questionnaire.

\begin{tabular}{|c|c|c|c|c|c|}
\hline \multirow{2}{*}{ Dependent variable } & \multicolumn{2}{|c|}{ Baseline } & \multicolumn{2}{|c|}{ Time 2} & \multirow{2}{*}{$P$} \\
\hline & Mean & $S D$ & Mean & $S D$ & \\
\hline \multicolumn{6}{|l|}{ SIAQ } \\
\hline Skill & 5.62 & 0.86 & 5.97 & 0.74 & 0.04 \\
\hline Strategy & 5.24 & 0.99 & 5.65 & 0.79 & 0.07 \\
\hline Goals & 5.77 & 1.00 & 6.20 & 0.67 & 0.00 \\
\hline Affect & 6.02 & 0.94 & 6.38 & 0.76 & 0.16 \\
\hline Mastery & 5.14 & 1.20 & 5.72 & 0.69 & 0.01 \\
\hline \multicolumn{6}{|l|}{ Practice } \\
\hline Goal Setting & 3.43 & 1.01 & 3.67 & 0.77 & 0.26 \\
\hline Emotional Control & 3.55 & 0.60 & 3.65 & 0.64 & 0.46 \\
\hline Automaticity & 3.51 & 0.64 & 3.84 & 0.48 & 0.04 \\
\hline Relaxation & 2.71 & 0.96 & 3.65 & 0.93 & 0.00 \\
\hline Self-Talk & 3.48 & 0.75 & 3.93 & 0.02 & 0.01 \\
\hline Imagery & 3.36 & 0.90 & 3.82 & 0.73 & 0.04 \\
\hline Attentional Control & 3.47 & 0.60 & 3.71 & 0.43 & 0.18 \\
\hline Activation & 3.44 & 0.60 & 3.65 & 0.65 & 0.16 \\
\hline \multicolumn{6}{|l|}{ Competition } \\
\hline Goal Setting & 3.58 & 0.93 & 3.99 & 0.58 & 0.06 \\
\hline Emotional Control & 3.43 & 0.28 & 3.40 & 0.44 & 0.18 \\
\hline Automaticity & 2.91 & $\mathbf{0 . 8 3}$ & 3.43 & $\mathbf{0 . 8 1}$ & 0.01 \\
\hline Relaxation & 3.48 & 0.83 & 3.79 & 0.69 & 0.06 \\
\hline Self-Talk & 3.51 & 0.92 & 3.95 & 0.71 & 0.04 \\
\hline Imagery & 3.50 & 0.77 & 4.02 & 0.58 & 0.02 \\
\hline Negative Thinking & 3.74 & 0.71 & 4.01 & 0.66 & 0.05 \\
\hline Activation & 3.43 & 0.69 & 3.79 & 0.62 & 0.04 \\
\hline
\end{tabular}

Note: Bolded variables were significant.

A 2 (time) $\times 1$ (group) ANOVA indicated a significant mean difference between baseline and Time 2 for mastery imagery $F(1,21)=7.49, P=0.01, \beta=0.74$. Mean scores indicate that participants mastery imagery ability improved from baseline $(m=5.14)$ to time 2 ( $m$ $=5.72$ ). There was not a significant difference across time for strategy $(P=0.07)$ or Affect $(P=0.16)$ imagery ability.

To assess the effectiveness of the protocol repeated measures ANOVAs were run for each subscale. The 2 (time) $\times 1$ (group) ANOVA indicated a significant mean difference between baseline and Time 2 for practice automaticity $F(1,19)=4.85, P=0.04, \beta=$ 0.55 . Mean scores indicate that participants used automaticity skills more at time $2(\mathrm{~m}=3.84)$ than at baseline $(m=3.51)$. The 2 (time) $\times 1$ (group) ANOVA's indicated a significant mean difference between baseline and Time 2 for practice relaxation $F$ $(1,19)=14.94, P=0.00, \beta=0.96$. Mean scores indicate that participants used relaxation skills during practice more at time $2(m=3.65)$ than at baseline $(m=$ 2.71). The 2 (time) $\times 1$ (group) ANOVA's indicated a significant mean difference between baseline and Time 2 for practice self-talk $F(1,19)=6.80, P=0.02, \beta=$ 0.70 . Mean scores indicate that participants used self-talk at practice more at time $2(m=3.93)$ than at baseline $(\mathrm{m}=3.48)$. The 2 (time) $\times 1$ (group) ANOVA's indicated a significant mean difference between baseline and Time 2 for practice imagery $F(1$, 19) $=4.84, P=0.04, \beta=0.55$. Mean scores indicate that participants used imagery more at time $2(\mathrm{~m}=3.36)$ than at baseline $(\mathrm{m}=3.82)$. There were no significant changes from baseline to Time 2 for goal setting $(P=$ 0.26), emotional control $(P=0.46)$, attentional control 
( $P=0.18)$ or activation $(P=0.16)$ use during practice.

The 2 (time) $\times 1$ (group) ANOVA's indicated a significant mean difference between baseline and Time 2 for competition automaticity $F(1,18)=8.72, P=$ $0.01, \beta=0.80$. Mean scores indicate that participants believed they would use automaticity skills more at time $2(m=2.91)$ than at baseline $(m=3.43)$. The 2 (time) $\times 1$ (group) ANOVA's indicated a significant mean difference between baseline and Time 2 for self-talk during competition $F(1,19)=4.81, P=0.04$, $c .55$. Mean scores indicate that participants believed they would use self-talk more at time $2(m=3.95)$ than at baseline $(m=3.51)$. The 2 (time) $\times 1$ (group) ANOVA's indicated a significant mean difference between baseline and Time 2 for imagery use during competition $F(1,18)=7.02, P=0.02, \beta=0.71$. Mean scores indicate that participants believed they would use imagery more at time $2(m=4.02)$ than at baseline $(m=3.50)$. The 2 (time) $\times 1$ (group) ANOVA's indicated a significant mean difference between baseline and Time 2 for negative thinking during competition $F(1,18)=4.36, P=0.05, \beta=0.51$. Mean scores indicate that participants believed they would use less negative thinking (indicated by a higher mean) at time $2(m=4.01)$ than at baseline $(m=3.74)$. The 2 (time) $\times 1$ (group) ANOVA's indicated a significant mean difference between baseline and Time 2 for activation use during competition $F(1,18)=5.13, P=$ $0.04, \beta=0.57$. Mean scores indicate that participants believed they would use imagery more at time $2(\mathrm{~m}=$ 3.79) than at baseline $(m=3.43)$. There were no significant changes from baseline to Time 2 for goal setting $(P=0.06)$, emotional control $(P=0.18)$, or relaxation $(P=0.06)$ skills they felt they would use during competition.

\section{Discussion}

As this is a preliminary assessment of a novel sport psychology technique, the authors feel it is appropriate to include some of the anecdotal evidence and experiences of the sport psychology intervention team, coaches and athletes. This program was time intensive for the intervention team, and did require a small amount of invested time from the players. As this was the first IAVR program, the intervention team spent many hours working out the logistics of the program. However, the team feels that it would take significantly less time in the future now that the logistics have been worked out. Even with the time commitment, people on the intervention team firmly believe that the time was well worth it as they relate to the outcomes for the players. Anecdotally, the athletes reported enjoying the VR training, and were excited about using it for the upcoming season, and seasons to follow. Athletes reported that they found practicing imagery a lot easier than when initially starting the program. Several athletes commented on the fact that they were now using imagery outside of the program, and that they felt more comfortable with it due to the IAVR program. The athletes requested several other filming views for future filming, indicating their intent to continue with the VR program. One of the coaches on the team gave the following assessment of the program "After using the VR program I noticed several improvements in our players. Players were better able to visualize mechanical adjustments to their swing. Before the use of the program, players would be told how to make a mechanical adjustment but would often have trouble visualizing what the adjustment entailed. After use of the program, players were better able to understand what adjustment needed to be made because they had practiced with the VR goggles. I believe that the VR program helped increase confidence in our players particularly in freshman who were able to get in more reps with the VR than they would have otherwise. I believe this is a very beneficial program, and anyone who trains with it properly can increase imagery ability, confidence and their overall performance. (Assistant Coach)."

These sentiments were mimicked in that of a player who stated "The VR program is great for any ball player at any level. For me being a freshman at the 
division one level I was a little over eager to succeed right away. I was playing faster and harder than ever, and trying to impress my new team. The VR program was very beneficial for me, I was able to get extra reps mentally in my dorm room while I wasn't near the baseball field. Not only is it convenient but it also helped me to relax and be calm in the box. The program also gave me some confidence because I watched myself succeed so many times. I used the VR two to three times a day. Each time I would watch my video three times in a row, so by the end of the day I would have watched my entire video six to nine times. If I could change one thing it would have been to get more video. I truly believe this program helped me get ready for upcoming season and I would recommend it to any athlete in any sport (Athlete)."

The inferential statistical analysis shed some light on to the effectiveness of an IAVR protocol, however these results should be seen as a jumping off point for more research opposed to conclusive findings. As this is a novel psychological strategy, it is important that more research is done before any conclusions can be made. That said, results indicated that imagery ability was improved for three of the five subscales. This is a promising finding that indicates that IAVR could help athletes improve their imagery ability and therefore their likelihood of using imagery effectively. Future research should assess whether an IAVR program can affect all five subscales of imagery ability if it specifically targets all areas. For this intervention, each individual athlete had their own areas targeted (i.e., one person may have had responses related to affect, another to mastery). Although preliminary these results are very promising, especially considering the importance of imagery ability for an athlete to achieve the many benefits of imagery use.

The TOPS analysis indicated several psychological skills and strategies were used more after the IAVR protocol was completed than at baseline. Imagery use was increased which is expected as it was a component of the study, and with imagery ability increases come imagery use increases [27]. Along with the increase in imagery use were increases in positive self-talk, and automaticity in both practice and competition. It makes sense that these skills and strategies would be increased as the VR allowed for players to develop a sense of automaticity through repetition by watching themselves in the task over and over again. Each athlete also had a component of their script in which it would refer to a cue word that athlete used to calm down (self-talk strategy), therefore both of these strategies were a focus of the intervention and it therefore makes sense that they both increased in practice and competition. Interestingly, relaxation was significantly increased in practice but not competition. The most common response propositions built into the imagery scripts related to feeling calm and relaxed (relaxation strategy), or feeling confident. It may be that athletes perceived the relaxation to be more useful in practice, conversely, activation use went up significantly in competition but not practice. This may be that athletes see competition as the time to be "activated" as opposed to practice, however more research would need to assess these findings before any conclusions can be made. Negative thinking during competition was decreased (indicated by a higher mean due to reverse coding of the subscale), indicating that the protocol had a positive impact on how a person thinks about the good and the bad of competition.

Like any new intervention there were several issues to figure out along the way. Specifically, figuring out the filming protocol was time intensive and challenging. The team seemed to buy in automatically, however, 5 participants did not participate. It was also not available for players who were injured during the time of filming. A limitation specific to pitchers was getting the film of the pitchers while staying within their fall pitch count. At the beginning of the intervention, three athletes complained that the goggles made them feel dizzy/nauseous, however, they all kept using the goggles and found that this feeling went away after a few uses. In the future, it is recommend that 
IAVR be done on an individual basis for players who are interested in the program opposed to an entire team. This would drastically reduce the main limitation of time invested on the part of the protocol team. That said having 22/27 participants follow a sport psychology protocol was in the eyes of those involved, quite successful. This study also highlights several unanswered questions on the use of VR and IAVR protocols in sport. Future research should investigate how VR works for psychological and physical skill development with imagery versus without imagery. The baseball team used for this study had been introduced to imagery as part of their training program before this study started. Future research should assess whether or not IAVR is effective in teaching imagery to those who have never used it previously, and to those who have poor imagery ability at baseline. Furthermore, IAVR needs to be assessed through experimental means, particularly those focused on assessing not just whether or not it is effective, but also why it is effective. Just as there is research on the Who, What, When, Where, Why's and How's of imagery, research should address these same questions in relation to VR research in general and IAVR specifically.

With the constant changes in VR technology, it is expected that this technology will become easier and easier to use, making this a potentially viable and important tool for sport psychology consultants to consider when working with athletes. Like other technological tools, it should only be used when it adds to the athletes' experiences, and by people who truly understand how to develop the program and utilize it for maximum gain. Overall, this applied work indicates that an Imagery Assisted Virtual Reality protocol may be an effective, novel and affordable tool for sport psychology consultants to use with their athletes.

\section{Acknowledgement}

The authors would like to acknowledge Alexa Knuth, Molly Josephs, and Corinne Brent for their help with this research. We also want to thank Logan Anderson for his commitment to this project.

\section{References}

[1] Smith, D. 2015. "One of the NFL's Top Quarterbacks Trains with Virtual Reality.” Business Insider. Accessed January 12, 2016. http://www.businessinsider.com/nfl-virtual-reality-201511

[2] Zorowitz, J. 2015. "It Just Got Real." NBC Sports. Accessed January $10,2016$. http://sportsworld.nbcsports.com/virtual-reality-sports-ar kansas-kentucky/

[3] Apstein, S. 2015. "Sports Illustrated's Innovation of the Year: Virtual Reality." Sports Illustrated. Accessed January $10,2016$. http://www.si.com/sportsperson/2015/12/18/innovation-o f-the-year-virtual-reality

[4] Chafkin, M. 2015. "Why Facebook's \$2 Billion Bet on Oculus Rift Might One Day Connect Everyone on Earth.” Vanity Fair Hive. Accessed January 11, 2016 http://www.vanityfair.com/news/2015/09/oculus-rift-mar k-zuckerberg-cover-story-palmer-luckey

[5] Bleacher Report. 2015. "New Virtual Reality System Is Changing Sports Forever." Bleacher report. Accessed January 12, 2016. http://bleacherreport.com/articles/2563010-stanfords-new -virtual-reality-system-is-changing-sports-forever

[6] Halton, J. 2008. "Virtual Rehabilitation with Video Games: A New Frontier for Occupational Therapy." Occupational Therapy Now 9 (6): 12-4.

[7] Chen, C. H., Jeng, M. C., Fung, C. P., Doong, J. L., and Chuang, T. Y. 2009. "Psychological Benefits of Virtual Reality for Patients in Rehabilitation Therapy." Journal of Sport Rehabilitation 18 (2): 258-68.

[8] Golomb, M. R., Warden, S. J., Fess, E., Rabin, B., Yonkman, J., Shirley, B., and Burdea, G. C. 2011. "Maintained Hand Function and Forearm Bone Health 14 Months after an In-Home Virtual-Reality Videogame Hand Telerehabilitation Intervention in an Adolescent with Hemiplegic Cerebral Palsy." Journal of Child Neurology 26 (3): 389-93.

[9] Deutsch, J. E. 2011. "Using Virtual Reality to Improve Walking Post-Stroke: Translation to Individuals with Diabetes." Journal of Diabetes Science and Technology 5 (2): 309-14.

[10] Jack, D., Boian, R., Merians, A. S., Tremaine, M., Burdea, G. C., Adamovich, S. V., and Poizner, H. 2001. "Virtual Reality-Enhanced Stroke Rehabilitation." IEEE Transactions on Neural Systems and Rehabilitation Engineering 9 (3): 308-18. 
[11] Mirelman, A., Maidan, I., and Deutsch, J. E. 2013. "Virtual Reality and Motor Imagery: Promising Tools for Assessment and Therapy in Parkinson's Disease." Movement Disorders 28 (11): 1597-608.

[12] Wiederhold, B. K., Soomro, A., Riva, G., and Wiederhold, M. D. 2014. "Future Directions: Advances and Implications of Virtual Environments Designed for Pain Management." Cyberpsychology, Behavior, and Social Networking 17 (6): 414-22.

[13] Difede, J., Cukor, J., Jayasinghe, N., Patt, I., Jedel, S., Spielman, L., and Hoffman, H. G. 2007. "Virtual Reality Exposure Therapy for the Treatment of Posttraumatic Stress Disorder Following September 11, 2001.” Journal of Clinical Psychiatry 68 (11): 1639-47.

[14] Watson, G., Brault, S., Kulpa, R., Bideau, B., Butterfield, J., and Craig, C. 2011. "Judging the 'Passability' of Dynamic Gaps in a Virtual Rugby Environment." Human Movement Science 30 (5): 942-56.

[15] Petit, J. P., and Ripoll, H. 2008. "Scene Perception and Decision Making in Sport Simulation: A Masked Priming Investigation." International Journal of Sport Psychology 39 (1): 1-19.

[16] Bideau, B., Kulpa, R., Ménardais, S., Fradet, L., Multon, F., Delamarche, P., and Arnaldi, B. 2003. "Real Handball Goalkeeper vs. Virtual Handball Thrower.” Presence 12 (4): 411-21.

[17] Levy, R., and Katz, L. 2007. "Virtual Reality Simulation: Bobsled and Luge." In Proceeding of 6th International Symposium of the International Association of Computer Science in Sport, 241-51.

[18] Fels, S., Kinoshita, Y., Chen, T. P. G., Takama, Y., Yohanan, S., Gadd, A., and Funahashi, K. 2005. "Swimming Across the Pacific: A VR Swimming Interface." IEEE Computer Graphics and Applications 25 (1): 24-31.

[19] Chuang, T. Y., Chen, C. H., Chang, H. A., Lee, H. C., Chou, C. L., and Doong, J. L. 2003. "Virtual Reality Serves as a Support Technology in Cardiopulmonary Exercise Testing." Presence: Teleoperators and Virtual Environments 12 (3): 326-31.

[20] Jeannerod, M. 1995. "Mental Imagery in the Motor Context." Neuropsychologia 33 (11): 1419-32.

[21] Holmes, P. S., and Collins, D. 2001. "The PETTLEP Approach to Motor Imagery: A Functional Equivalence Model for Sport Psychology." Journal of Applied Sport Psychology 13 (1): 60-83.

[22] Smith, D., Wright, C., Allsopp, A., and Westhead, H. 2007. "It's All in the Mind: PETTLETP-Based Imagery and Sports Performance." Journal of Applied Sport Psychology 19 (1): 80-92.

[23] Wakefield, C., and Smith, D. 2012. "Perfecting Practice:
Applying the PETTLEP Model of Motor Imagery." Journal of Sport Psychology in Action 3 (1): 1-11.

[24] Holmes, P. S., and Camels, C. 2010. "A Neuroscientific Review of Imagery and Observation Use in Sport." Journal of Motor Behavior 40 (5): 433-45.

[25] Williams. S. E., and Cumming, J. 2012. “Athletes' Ease of Imaging Predicts Their Imagery and Observational Learning Use." Psychology of Sport and Exercise 13 (4): 362-70.

[26] Morris, T., Spittle, M., and Watt, A. P. 2005. "Imagery in Sport." Champaign, Illinois: Human Kinetics.

[27] Williams. S. E., and Cumming, J. 2011. "Development and Validation of the Sport Imagery Ability Questionnaire." Journal of Sport and Exercise Psychology 33 (3): 416-40.

[28] Thomas, P. R., Murphy, S. M. and Hardy, L. 1999. "Test of Performance Strategies: Development and Preliminary Validation of a Comprehensive Measure of Athletes' Psychological Skills." Journal of Sports Sciences 17 (9): 697-711.

[29] White, A., and Hardy, C. 1998. "An In-Depth Analysis of the Uses of Imagery by High-Level Slalom Canoeists and Artistic Gymnasts." The Sport Psychologist 12 (4): 387-403.

[30] Feltz, D. L., Short, S. E., and Sullivan, P. J. 2008. Self-Efficacy in Sport. Champaign, IL: Human Kinetics.

[31] Short, S. E., and Ross-Stewart, L. 2008. "A Review of Self-Efficacy Based Interventions." In Applied Sport Psychology Advances: A Review, edited by Mellalieu, S., and Hanton, S. London, UK: Routledge.

[32] Martinent, G., Ledos, S., Ferrand, C., Campo, M., and Nicolas, M. 2015. “Athletes' Regulation of Emotions Experienced During Competition: A Naturalistic Video-Assisted Study." Sport, Exercise, and Performance Psychology 4 (3): 188-205.

[33] Robin, N., Dominique, L., Toussaint, L., Blandin, Y., Guillot, A., and Her, M. L. 2007. "Effects of Motor Imagery Training on Service Return Accuracy in Tennis: The Role of Imagery Ability." International Journal of Sport and Exercise Psychology 5 (2): 175-86.

[34] Williams, S. E., Cooley, S. J., Newell, E., Weibull, F., and Cumming, J. 2013. "Seeing the Difference: Advice for Developing Effective Imagery Scripts for Athletes." Journal of Sport Psychology in Action 4 (2): 109-21.

[35] Cumming, J., and Ramsey, R. 2008. "Imagery Interventions in Sport." In Applied Sport Psychology Advances: A Review, edited by Mellalieu, S., and Hanton, S. London, UK: Routledge.

[36] Driskell, J. E., Copper, C., and Moran, A. 1994. "Does Mental Practice Enhance Performance?” Journal of Applied Psychology 79 (4): 481-92. 